\title{
Generation of Attributes for Bangla Words for Universal Networking Language(UNL)
}

\author{
Muhammad Firoz Mridha \\ Dept. of Computer Science \\ Stamford University Bangladesh \\ Dhaka, Bangladesh
}

\author{
Manoj Banik \\ Dept. of Computer Science and Engineering \\ Ahsanullah University of Science and Technology, \\ Dhaka, Bangladesh
}

\author{
Kamruddin Md. Nur \\ Dept. of Computer Science \\ Stamford University Bangladesh \\ Dhaka, Bangladesh
}

\author{
Mohammad Nurul Huda \\ Dept. of Computer Science and Engineering \\ United International University \\ Dhaka, Bangladesh
}

\begin{abstract}
The usage of native language through Internet is highly demanding now a day due to rapidly increase of Internet based application in daily needs. It is important to read all information in Bangla from the internet. Universal Networking Language (UNL) addressed this issue in most of languages. It helps to overcome the language barrier among people of different nations to solve problems emerging from current globalization trends and geopolitical interdependence. In this paper we propose a work that aims to contribute with morphological analysis of those Bangla words from which we obtain roots and Primary suffixes and developing of grammatical attributes for roots and Primary suffixes that can be used to prepare Bangla word dictionary and Enconversion/Deconversion rules for Natural Language Processing(NLP).
\end{abstract}

Keywords- Morphology; Bangla Roots; Primary Suffix; Grammatical Attributes; Universal Networking Language.

\section{INTRODUCTION}

In the last few years, machine translation techniques have been applied to web environments. The growing amount of available multilingual information on the Internet and the Internet users has led to a justifiable interest on this area. Hundreds of millions of people of almost all levels of education and attitudes, of different jobs all over the world use the Internet different purposes [1]. English is the main language of the Internet. Understandably, not all people know English. Urgent need, therefore, arose to develop interlingua translation programs. The main goal of the UNL system is to provide Internet users access to multilingual websites using a common representation. This will allow users to visualize websites in their native languages. The UNL system has a growing relevance since the usage of the WWW is generalized across cultural and linguistic barriers. Many languages [10] such as Arabic, French, Russian, Spanish, Italian, English, Chinese or Brazilian Portuguese have already been included in the UNL platform. Lexical knowledge representation is a critical issue in natural language processing systems. Recently, the development of large-scale lexica with specific formats capable of being used by several different kinds of applications has been given special focus; in particular to multilingual systems. Our aim is to introduce Bangla into this system. In order to implement this project with the lowest time and human effort costs, we will reuse linguistic resources already available as much as possible.

In this paper we present the UNL system for Bangla. The major components of our research works touches upon i) development of grammatical attributes for Bangla root and Krit Prottoy to construct Bangla Word Dictionary and use of morphological analysis ii) UNL Expression of the Bangla attributes and iii) Selecting scheme of attributes. In section 2 we describe the UNL system. In sections 3, 4 and 5 we present our main works that include all the above three components.

\section{UNIVERSAL NETWORKING LANGUAGE}

UNL is an artificial language that allows the processing of information across linguistic barriers [10]. This artificial language has been developed to convey linguistic expressions of natural languages for machine translation purposes. Such information is expressed in an unambiguous way through a semantic network with hyper-nodes. Nodes (that represent concepts) and arcs (that represent relations between concepts) compose the network. UNL contains three main elements:

- Universal Words: Nodes that represent word meaning.

- Relation Labels: Tags that represent the relationship between Universal Words. Tags are the arcs of UNL hypergraph.

- Attribute Labels: Additional information about the universal words.

These elements are combined in order to establish a hierarchical Knowledge Base (UNLKB) [10] that defines unambiguously the semantics of UWs. The UNL Development Set provides tools that enable the semi-automatic conversion of natural language into UNL and vice-versa. Two of such tools are the EnConverter and the DeConverter. The main role of EnConverter [11] is to translate natural language sentences into UNL expressions. This tool implements a language 
independent parser that provides a framework for morphological, syntactic and semantic analysis synchronously. This allows morphological and syntactical ambiguities resolution. The DeConverter [3, 12], on the other hand, is a language independent generator that converts UNL expressions to natural language sentences.

\section{A. Universal Words}

Universal Words are words that constitute the vocabulary of UNL. A UW is not only a unit of the UNL syntactically and semantically for expressing a concept, but also a basic element for constructing a UNL expression of a sentence or a compound concept. Such a UW is represented as a node in a hypergraph. There are two classes of UWs from the viewpoint in the composition:

- labels defined to express unit concepts and called "UWs" (Universal Words)

- a compound structure of a set of binary relations grouped together and called "Compound UWs".

\section{B. Relational Labels}

The relation [1] between UWs is binary that have different labels according to the different roles they play. A relation label is represented as strings of three characters or less. There are many factors to be considered in choosing an inventory of relations. The following is an example of relation defined according to the above principles.

Relation: There are 46 types of relations in UNL. For example, agt (agent), agt defines a thing that initiates an action, agt(do, thing), agt(action, thing), obj(thing with attributes) etc.

\section{Attributes}

The attributes represent the grammatical properties of the words. Attributes of UWs are used to describe subjectivity of sentences. They show what is said from the speaker's point of view: how the speaker views what is said. This includes phenomena technically $[4,5]$ called speech, acts, propositional attitudes, truth values, etc. Conceptual relations and UWs are used to describe objectivity of sentences. Attributes of UWs enrich this description with more information about how the speaker views these state of affairs and his attitudes toward them.

\section{MORPHOLOGY OF BANGLA WORDS}

Morphology is the field of linguistics that studies the structure of words. It focuses on patterns of word formation within and across languages, and attempts to formulate rules that model the knowledge of the speakers of those languages. Thus morphological analysis is found to be centered on analysis and generation of word forms. It deals with the internal structure of words and how words can be formed. Morphology plays an important[2, 8] role in applications such as spell checking, electronic dictionary interfacing and information retrieving systems, where it is important that words that are only morphological variants of each other are identified and treated similarly. In natural language processing (NLP) and machine translation (MT) systems we need to identify words in texts in order to determine their syntactic and semantic properties [7]. Morphological study comes here to help with rules for analyzing the structure and formation of the words. A Bangla morpheme, besides the root word, is supposed to be represented in the Bangla-UNL dictionary using the following UNL format [10].

[HW] "UW" (ATTRIBUTE 1, ATTRIBUTE 2 ..) $<$ FLG, FRE, PRI>

$\mathrm{HW} \leftarrow$ Head Word (Bangla Word)

$\mathrm{UW} \leftarrow$ Universal Word

ATTRIBUTE $\leftarrow$ Attribute of the HW

FLG $\leftarrow$ Language Flag

FRE $\leftarrow$ Frequency of Head Word

$\mathrm{PRI} \leftarrow$ Priority of Head Word

The attributes describe the nature of the head word classifying it as a grammatical, semantic or morphological feature. So, we will be especially concerned about representation of morphemes using various attributes.

\section{A. Prefixes (উभসর্গ)}

Prefixes are the words that are used before words to express various meanings of the same words. There are around fifty (50) prefixes used in Bangla sentences. In Shangskrit Bangla we use twenty (20) prefixes[2] say , প্র(প্রকর্ম), গরা(বৈপরীত্য), অগ(বৈপরীত্য) etc. , in Bangla we use thirteen prefixes (13) prefixes[2] such as বে(বৈপরীত্য), গর(বৈপরীত্য), অন(অভাব) etc., five(5) foreign prefixes[5] such as গর (না),বদ (খারাগ) etc., four English prefixes[14] such as সাব(অধীন অর্থে), হেড(প্রধান), ফুল(পুরা), হাফ(অর্ধ) etc. These prefixes are used before words to make thousands of meaningful Bangla Words.

In our work, we will make separately Word Dictionary entries for all of these prefixes and words, so that they can combinely make meaningful words by applying rules. For example, if we consider prefix “প্রতি'[9] (means like/similar/every/opposite/against etc.) we can make “প্রতিদিন”, “প্রতিশব্দ" etc. Now we can make the word "প্রতি" for dictionary entry. But the word "প্রতি" has two or more meanings so that we have to represent two or more dictionary entries for the word as follows.

$$
\begin{aligned}
& \text { [প্রতি] }\{\} \text { "every (icl>thing)" (ABSTRACT THING) }<\mathrm{B}, 0,0> \\
& {[\text { প্রতি }]\{\} \text { "opposite (icl>thing)" (ABSTRACT THING) }} \\
& <\mathrm{B}, 0,0>
\end{aligned}
$$

Now if we want to represent the concepts of the words say প্রতিদিন, প্রতিশব্দ etc., we need not represent the whole words. We have to represent only the words “দিन", “শব্দ" in the dictionary entry as per the following format.

[দিন] \{\} "day (icl>period>time)" (N, ABSTRACT THING, LIGHT) $<\mathrm{B}, 0,0\rangle$

[শব্দ] \{\} "sound (icl>occure $>$ thing)" (N, ABSTRACT THING) $<\mathrm{B}, 0,0\rangle$ 


\section{B. Noun Morphology}

Bangla Nouns have very strong and structural inflectional morphology base on case. Case of noun may be nominative ("ছেেলে", boy), accusative (ছেলে-কে"), to the boy) and genetive (“ছেলে-র”, of the boy) and so on. Gender and number are also important for identifying proper categories of nouns. Number may be singular ("ছেলে", boy or "ছেলেটি', the boy, “বই”, book,

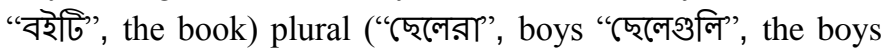
“বইগুলো”, the books etc.). So, from the word “ছেলে’ we get "ছেেলের", "ছেলেকে", "ছেলেটি,, "ছেলেগুলি” etc. and from the word “বই” we get “বইটি', “বইগুলি’ etc. Some dictionary entries may look like. [ছেলে] \{\} "boy (icl>person)" (N, HN, C, ANI) $<\mathrm{B}, 0,0\rangle$

Here, "boy (icl>person)" is the UW for "ছেলে" but "র", “কে" etc. have no UWs. Therefore, they should be represented in the dictionary only with grammatical attributes as follows.

[ส] \{\} “" $(3 \mathrm{P}, \mathrm{SUF}, \mathrm{N})<\mathrm{B}, 0,0>$

[কে] \{\} “"” (3P, SUF, N, HUMN, SG) $<\mathrm{B}, 0,0>$

[สा] \{\} “" (3P, PL, SUF, N, HUMN) $<\mathrm{B}, 0,0>$

[টি] \{\} “" (N, SG, SUF,3P) $<\mathrm{B}, 0,0>$

[গুলি] \{\}$“ "(\mathrm{~N}, \mathrm{PL}, \mathrm{SUF}, 3 \mathrm{P})(<\mathrm{B}, 0,0>$

\section{Adjective Morphology}

As Adjective we can consider Bangla words "সাহস", “'সুন্দর” and "ভাল" meaning "bravery", "beautiful" and "good" in English respectively. From the first word we get সাহসী (সাহস+ই), সাহসের (সাহস+এর). And from the second and third words we get সুন্দরী, ভালোর, ভালোটা etc. We have to have the dictionary entries for সাহস, সুন্দর, ভালো, ই, এর, র, টা to make the meaningful words সাহসী, সাহসের, সুন্দরী, ভালোটা etc. by combining the morphemes with the root words using analysis rules.

\section{Pronoun Morphology}

Here we can consider the word root “তাহা'(he/she). From this we get তাহা-রা, তাহা-কে, তাহা-দের, তাহা-দের-কে, তাহাদিগকে etc. So, we have to consider these morphemes রা, কে, দের, দিগকে for dictionary entries to form words with “তাহা” as above.

\section{E. Verb Morphology}

Diversity of verb morphology in Bangla is very significant. We can select the head words as the Longest Common Lexical Unit (LCLU) of all the possible transformations of the word [8].

We can give the example of the Bangla word "भড়" (means read). The corresponding UW in basic form is "read". The dictionary entry is: [भড়া] \{ $\}$ "read (icl $>$ do)", where 'भড় \&' is the head word and (icl>do) is from the knowledge base. Some possible transformations of "भড়" in the Bangla to UNL dictionary are given as follows $[9,10]$ :
If we consider 'भড়' (means read) as a root, we can represent this root in the dictionary as

[भড়] \{\} “read (icl>do)” (V, @present)<B,0,0>

Some transformations based on the persons and tenses are.

For first person:

[भড়] \{ \} "read (icl>do)" (ROOT, BANJANT)<B, 0, 0>

[ই] \{ $\}$ "read (icl $>$ do)" (ROOT, BANJANT, PRESENT INDEF) $<$ B, 0, 0>

[ইতেছ্ছি \{\} "read (icl>do)" (ROOT, BANJANT, PRESENT CONT) $\langle\mathrm{B}, 0,0\rangle$

For second person:

[भড়] \{ \} "read (icl>do)" (ROOT, BANJANT)<B, 0, 0>

[ইতেছ্] \{\} "read (icl>do)" (ROOT, BANJANT, PRESENT CONT) $<\mathrm{B}, 0,0>$

[ইবে] \{ \} "read (icl $>$ do)" (ROOT, BANJANT, FUTURE INDEF) $<\mathrm{B}, 0,0>$

For third person:

[भড়] \{\} "read (icl>do)" (ROOT, BANJANT)<B, 0, 0> [ইয়াছ্ছে] \{ \} "read (icl>do)" (ROOT, BANJANT, PRESENT PERF) $\langle\mathrm{B}, 0,0\rangle$

[ইতেছ্ছে] \{ \} "read (icl>do)" (ROOT, BANJANT, PRESENT CONT) $<\mathrm{B}, 0,0>$

For resolving the ambiguities of the words গিয়েছি, গিয়েছ্ছিলাম, গিয়েছ্নেন, গিয়েছ্ছিলেন, যাইতে থাকবে, etc. we have to define them as full words for dictionary entries. For instance [গিয়েছিলাম] \{\} “go (icl>move>do)"(V, PAST, INDEF, 1P). Using the same procedure we can make dictionary entries for different transformations of other roots such as কর (do), লিথ (write),দে (give) etc.

\section{MORPHOLOGY OF BANGLA ROOT WORDS}

Bangla Language contains a lot of verbs. The core part of those verbs is called roots. In another way if we split the verbs we get two parts Roots and Suffixes. From verbs if we remove suffixes we get roots. For example 'Kłi' (do) is a verb. Its two parts are: $\mathrm{Ki}+\mathrm{G}$; here ' $\mathrm{Ki}$ ' is a root and ' $\mathrm{G}$ ' is a suffix. Some other Bangla roots verbs are Pj\&, co\&, ai\&, Mo\&, NI\&, bvP\&, Kuv'\& etc.

\section{A. Bangla Primary Suffixes (K... cÖZ"q)}

We know that the core of the verb is called root and if number of suffixes are added to roots then they form verbs. When sound or sounds [8] are added with roots and form nouns or adjectives then the root words are called root verbs and the sound or sounds are added with root verbs are called Primary Suffixes. For example $\mathrm{Pj} \&$ (Root verb)+Ab\& (Primary Suffix) $=P j b$ (Noun) and Pj\& (Root verb)+Aš- (Primary Suffix $)=$ Pjš - (Adjective). Some others primary suffixes are Ab, Abv, Awb, AK, Av etc. 


\section{B. Vowel ended and Consonant ended root}

Verb roots that are ended with vowel are called SORANTO root. For example, চा, হ, भा, नो, ধू, था, ছा etc. And verb roots that are ended with consonant are called BANJANTO root. For example, কর,,,চল्, ,গড়,নাচ, চিন্.etc.

\section{Morphological Analysis of Bangla verbs}

Morphological analysis is applied to identify the actual meaning of the word by identifying suffix or morpheme of that word. Every word is derived from a root word. A root word may have the different transformations. This happens because of different morphemes which are added with it as suffixes. So, the meaning of the word varies for its different transformations. For example, if we consider 'Ki\&'(do) as a root word then after adding Õ B' we get the word 'Kwi' $[6,8]$ which means a work done by some one(first person, present tense). Similarly after adding ' $A v$ ', we get the word 'Kiv'. Here, this word represents noun of the root word 'Ki\&'. Therefore, by morphological analysis we get the grammatical attributes of the main word. Derivational morphology is simple and a word rarely uses the derivational rule in more than two or three steps. The first step forms nouns or adjectives from verb roots. The next steps form new nouns and adjectives [5]. We have examined derivational morphology for UNL Bangla dictionary too.

\section{Methods of Finding Grammatical AtTRiButes}

Representing Universal Words (UWs) for each of the Bangla Head Word we need to develop grammatical attributes that describe how the words behave in a sentence. Grammatical Attributes (GA) have to be developed by the rules (Enconversion and Deconversion) and dictionary developers. They play very important rules for writing Enconversion and Deconversion rules because a rule uses GA in morphological and syntactic analysis, to connect or analyze one morpheme with another to build a meaningful (complete) word and to examine or define the position of a word in a sentence.

When we analyze the HWs for representing them in the word dictionary as UWs, we find all the possible specifications of the HWs as attributes named grammatical attributes, so that they can be used in the dictionary for making rules (EnCo and DeCo). For example, if we consider "crwLÓ meaning bird as a head word, then we can use attributes N (as it is noun), ANI (as bird is an animal), SG for singular number and CONCRETE (as it a concrete thing which is touchable).

So, this word can be represented in the dictionary as follows:

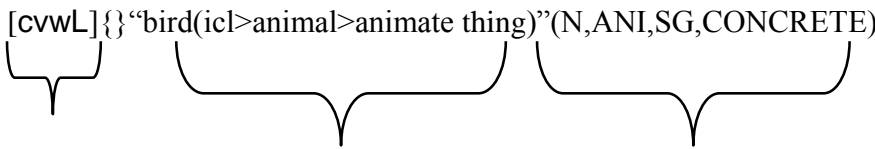

Head

Universal Word

Grammatical Attributes

Word

Same way we can represent the words avb (paddy), bvP\& (dance) etc. as follows:

[avb] \{\} "paddy(icl>plant>thing)"(N, PLANT, CONCRETE)

[bvP\&] \{\} "dance(icl>do)"(ROOT, BANJANT)
Where, $\mathrm{N}$ for noun, CONCRETE for any concrete thing which is touchable, ANI for animal and SG for singular number.

As we are the initiators of developing rules and word dictionary for Bangla we are proposing some grammatical attributes and their descriptions in table 1 .

\section{A. Primary suffixes with consonant ended roots}

(BANJANTO):

Some primary suffixes that are added with roots (which are ended with consonant) to form nouns and adjectives are given in table 2 .

Table 2 Consonant ended roots with primary suffixes

\begin{tabular}{|c|c|}
\hline Banjanto root + Primary suffix & Noun and Adjective \\
\hline কর্+অন্ত & করন্ত, \\
\hline পড়+অন্ত & পড়ন্ত, \\
\hline চল्+অন্ত & চলন্ত \\
\hline চল্+তি & চলতি \\
\hline পড়+তি & গড়তি \\
\hline কাট্+তি & কাটতি \\
\hline ঝুল্+তি & ঝুলতি \\
\hline
\end{tabular}

The dictionary entries for the roots and their suffixes are as follows:

[नाচ] \{\} "do (icl>do)"( ROOT, BANJANT)\

[भড়] \{\} "read (icl>do)"( ROOT, BANJANT)

[চল] \{\} "go (icl>do)"( ROOT, BANJANT)

[কাট़] \{\} "cut (icl>do)"( ROOT, BANJANT)

[পাকড় \{\} "attack (icl>do)"( ROOT, BANJANT)

[উড়] \{\} "fly (icl>do)"( ROOT, BANJANT)

[অन] \{\} “” (PROT, KPROT, BANJANT, MVNOUN)

[আ]\{\} “’” (PROT, KPROT, BANJANT, MNOUN)

[অন্ত] \{\} “” (PROT, KPROT, BANJANT, MADJ)

\section{B. Primary suffix with vowel ended roots (SORANTO):}

Some SORANTO is added only with primary suffix 'Av' to form nouns are shown in table 3 .

Table 3 Vowel ended roots with primary suffix

\begin{tabular}{|c|c|}
\hline Soranto root + Primary suffix & Noun \\
\hline চা+আ & চা 3য়া \\
\hline গা+আ & গাওয়া \\
\hline দে+আ & দে 3য়া \\
\hline থা+আ & খা 3য়া \\
\hline
\end{tabular}

So, the dictionary entries would be:

[চা]\{\} “want(icl>do)" ( ROOT, SORANT) $<\mathrm{B}, 0,0\rangle$ 
[भा] \{\} "get(icl>obtain)" ( ROOT, SORANT) <B,0,0>

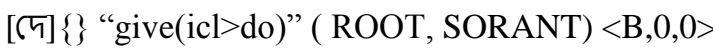

$[थ \mid]\{\}$ "eat(icl $>$ do)" ( ROOT, SORANT) $\langle\mathrm{B}, 0,0\rangle$

[आ] \{\} \}"” (PROT, KPROT, SORANT, MNOUN) <B, 0,0$\rangle$

$C$. There are some such primary suffixes which have two forms. One form is added with vowel ended roots and other forms are added with consonant ended roots. Those are given in table 4.

Table 4 Primary suffix with SORANTO roots and BANJANTO roots

\begin{tabular}{|c|c|}
\hline Banjanto root + Primary suffix & Noun \\
\hline চা+আ & চা ওয়া \\
\hline পা+আ & भाওয়া \\
\hline দে+আ & দে ওয়া \\
\hline চল्+আ & চলা \\
\hline পড়+আ & পড়া \\
\hline কাট়+আ & काটা \\
\hline
\end{tabular}

The UNL form:

[চा] \{\} "want(icl>do)" ( ROOT, SORANT) $\langle\mathrm{B}, 0,0\rangle$

[भा] \{\} "get(icl>obtain)" ( ROOT, SORANT) $\langle\mathrm{B}, 0,0\rangle$

$[$ দে \{\} "give(icl $>$ do)" ( ROOT, SORANT) $\langle\mathrm{B}, 0,0\rangle$

[আ] \{\}“"'(PROT,KPROT,SORANT,MNOUN) $\langle\mathrm{B}, 0,0\rangle$

[भড়] \{\} "read (icl>do)"( ROOT, BANJANT) $\langle\mathrm{B}, 0,0\rangle$

[চল]] \{\} "go (icl $>$ do)"( ROOT, BANJANT) $\langle\mathrm{B}, 0,0\rangle$

[কাট] \{\} "cut (icl $>$ do)"( ROOT, BANJANT) $<\mathrm{B}, 0,0\rangle$

[আ] \{\} “'”(PROT,KPROT,BANJANT,MNOUN) $<\mathrm{B}, 0,0>$

All the Roots are added with “আ” Krit Prottoy and form a meaningful word. For example,

$$
\begin{aligned}
& \text { কর্+আ= করা } \\
& \text { হার্+আ =হারা }
\end{aligned}
$$

But Some BANJANTA Roots (দूল,थুল) etc do not form a meaningful Bangla word to add with "আ" Prottoy. For example,

$$
\begin{aligned}
& \text { দूল्+আ=দুলা } \\
& \text { থूল+আ=2ুলा. }
\end{aligned}
$$

\begin{tabular}{|c|c|c|}
\hline $\begin{array}{l}\text { Grammatical } \\
\text { Attributes }\end{array}$ & Descriptions & $\begin{array}{l}\text { Examples (Here we } \\
\text { can use Bangla Words) }\end{array}$ \\
\hline ROOT & Only for verb root & $\begin{array}{l}\text { co\& (Read), Ki\&(do) } \\
\text { etc. }\end{array}$ \\
\hline SORANT & $\begin{array}{l}\text { Verb roots that are ended with } \\
\text { vowel }\end{array}$ & চা (want), যা(go) etc. \\
\hline BANJANT & $\begin{array}{l}\text { Verb roots that are ended with } \\
\text { consonant }\end{array}$ & $\begin{array}{l}\text { भড়(read), ai\& (catch) } \\
\text { etc. }\end{array}$ \\
\hline $1 \mathrm{P}$ & First person & vwg (I), Avgiv (we) etc. \\
\hline $2 \mathrm{P}$ & Second Person & Zzwg (you) etc. \\
\hline $3 \mathrm{P}$ & Third Person & $\begin{array}{l}\ddagger \mathrm{m} \text { cyi“l }(\mathrm{He}), \quad \ddagger \mathrm{m} \\
\text { gwnjv (She) etc. }\end{array}$ \\
\hline PROT & For all suffixes & $A v, A b, A v B$ etc. \\
\hline KPROT & $\begin{array}{l}\text { For the suffixes that are used } \\
\text { after roots to create Nouns, } \\
\text { Adjectives etc. }\end{array}$ & $\mathrm{BK}, \mathrm{Ab}$ etc. \\
\hline KBIVOKTI & $\begin{array}{l}\text { For the suffixes that are used } \\
\text { after roots to create only verbs. }\end{array}$ & $B, B \ddagger Z w Q$, †e etc. \\
\hline ADJ & For any adjective & $\begin{array}{l}\text { fvj (good), mys } \mathrm{i} \\
\text { (beautiful) etc. }\end{array}$ \\
\hline $\mathrm{N}$ & For any noun & $\begin{array}{l}\text { Kjg (pen), Avg(mango) } \\
\text { etc. }\end{array}$ \\
\hline MNOUN & $\begin{array}{l}\text { suffixes that are added with } \\
\text { roots to make nouns. }\end{array}$ & Av etc. \\
\hline MADJ & $\begin{array}{l}\text { suffixes that are added with } \\
\text { roots to make adjectives }\end{array}$ & Aš- etc. \\
\hline MVERB & $\begin{array}{l}\text { suffixes that are added with } \\
\text { roots to form verbs }\end{array}$ & $B, B \neq Z w Q$, †e etc. \\
\hline UROOT & For consonant ঊ & দুল, থুল etc. \\
\hline $\begin{array}{l}\text { PRESENT } \\
\text { INDEFINITE }\end{array}$ & $\begin{array}{l}\text { suffixes that create present } \\
\text { indefinite form of the sentence }\end{array}$ & B etc. \\
\hline HPRON & For human & আমি, সে etc. \\
\hline NUM & For number & $\varangle, ৭, ৯$ etc. \\
\hline NANI & For not amimate & বই, কলম etc. \\
\hline CONCRETE & For solidthing & জমি, ঘর etc. \\
\hline $\mathrm{HF}$ & For human food & ভাত, রুটি etc. \\
\hline FEMALE & For female person & সে(মহিলা) etc. \\
\hline BIV & For Bivokti & ইতেছে, ইয়াছে \\
\hline $\mathrm{V}$ & For Verb & করে,পড়ি etc \\
\hline $3 \mathrm{PG}$ & $3^{\text {rd }}$ Person General & করিতেছে, করিত etc \\
\hline IMPS & Imperative Sentence & করুক,করুন etc \\
\hline $3 \mathrm{PR}$ & $3^{\text {rd }}$ Person Respected & করিতেছ্নেন, করিবেন etc \\
\hline $2 \mathrm{PR}$ & $2^{\text {nd }}$ Person Respected & করিতেছ্নেন, করিবেন etc \\
\hline $2 \mathrm{PG}$ & $2^{\text {nd }}$ Person General & করিতেছ্, করিতেছিল etc \\
\hline $2 \mathrm{PN}$ & $2^{\text {nd }}$ Person Neglected & কর, করিস etc \\
\hline PAH & Past Habituate & করিত, করিতেন etc \\
\hline $1 \mathrm{P}$ & $1^{\text {st }}$ Person & করি, করিতেছি etc \\
\hline
\end{tabular}

Rather দোল in place of দুল and থোল in place of থুল is added with "আ" Krit Prottoy and form meaningful word. For example, দোল+আ=দোলা, (থোল +আ=(থালা.
Table 1 some proposed grammatical attributes

$D$. To solve this problem we divide BANJANTA Roots into two categories. One is General BANJANTA that is attributed as BANJNT and another is attributed with URoots. 
Table 5 Examples of general BANJANTO and URoots

\begin{tabular}{|c|c|}
\hline Banjanto root + Primary suffix & Noun \\
\hline কর্+আ & कরা \\
\hline भড়+আ & भড়া \\
\hline হার্+আ & হারা \\
\hline দুল্+আ & দুলা \\
\hline থুল+আ & থুলা \\
\hline দোল+আ & দোলা \\
\hline থোল+আ & থোলা \\
\hline
\end{tabular}

The UNL form:

[কর] \{\} "do (icl $>$ do)"( ROOT, BANJANT) $\langle\mathrm{B}, 0,0\rangle$

[হার] \{\} "lost (icl $>$ do)"( ROOT, BANJANT) $\langle\mathrm{B}, 0,0\rangle$

[আ]\{\} (PROT, KPROT, SORANT, MNOUN) $\langle\mathrm{B}, 0,0\rangle$

[দোল] \{\} "swing (icl $>$ do)"( ROOT, BANJANT) $\langle\mathrm{B}, 0,0\rangle$

[থোল] \{\} "open (icl $>$ do)"( ROOT, BANJANT) $\langle\mathrm{B}, 0,0\rangle$

[দूল] \{\} "swing(icl $>$ do)"( ROOT, BANJNT, URoot) $\langle\mathrm{B}, 0,0\rangle$

[थুল] \{\} “open(icl>do)"( ROOT, BANJNT, URoot ) $\langle\mathrm{B}, 0,0\rangle$

We make two entries in Bangla word dictionary for URoots. For example, দুল্ and দোল, থুল and থোল. In Bangla there are some primary suffixes which added with roots and form a new different words for example, বচ $+w^{3}=$ মू $w^{3}$ and मूচ $+w^{3}=$ মू $w^{3}$. These words are added to word dictionary in special category.

The dictionaries would be:

$\left[\mathrm{JW}^{3}\right]\{\}$ "speech (icl $>$ do)"( ROOT, BANJANT, SP) $\langle\mathrm{B}, 0,0\rangle$ $\left[\mathrm{DW}^{3}\right]\{\}$ "free (icl>do)"( ROOT, BANJANT, SP) $\langle\mathrm{B}, 0,0\rangle$

The suffixes আন্ত, তি, আ etc. will be in the dictionary only with grammatical attributes. They will be added with the roots to form verbs, nouns or adjectives using rules. In the above examples we have classified suffixes in the basis of adding either with SORANTO (vowel ended) or BANJANTO (consonant ended) to give them proper attributes so that they can be used to make appropriate rules enconversion and deconversion.

\section{CONCLUSION AND FUTURE WORK}

A system capable of understanding natural language sentences is of potentially unlimited uses in the field of natural language processing. In this paper we have generated grammatical attributes of Bangla roots and Krit Prottoy for developing Bangla Word Dictionary for Universal Networking Language (UNL).

We have presented some method to select grammatical attribute using morphological analysis Bangla words that can be used to make dictionary for converting the Bangla sentences to UNL documents and vice versa. We have done limited work so far for Bangla words.

Our future plan is to build a Bangla language server that will contain a complete Bangla Word Dictionary.

\section{REFERENCES}

[1] S. Abdel-Rahim, A.A. Libdeh, F. Sawalha, M. K. Odeh, "Universal Networking Language(UNL) a Means to Bridge the Digital Divide", Computer Technology Training and Indistrial Studies Center, Royal Scientific Sciety, March 2002.

[2] M. M. Asaduzzaman, M. M. Ali, "Morphological Analysis of Bangla Words for Automatic Machine Translation", International Conference on Computer, and Information Technology (ICCIT), Dhaka, 2003, pp.271276

[3] Serrasset Gilles, Boitel Christian, (1999) UNL-French econversion as Transfer \& Generation from an Interlingua with Possible Quality nhancement through Offline Human Interaction. Machine Translation Summit-VII, Singapore.

[4] M. E. H. Choudhury, M. N.Y. Ali, M.Z.H. Sarkar, R. Ahsan, "Bridging Bangla to Universal Networking Language- A Human Language Neutral Meta- Language", International Conference on Computer and Information Technology (ICCIT), Dhaka, 2005,pp.104- 109

[5] M.E.H. Choudhury, M.N.Y. Ali, "Framework for synthesis of Universal Networking Language", East West University Journal, Vol. 1, No. 2, 2008, pp. 28-43

[6] M.N.Y. Ali, J.K. Das, S.M. Abdullah Al Mamun, M. E.H. Choudhury, "Specific Features of a Converter of Web Documents from Bengali to Universal Networking Language", International Conference on Computer and Communication Engineering 2008(ICCCE'08), Kuala Lumpur, Malaysia.pp. 726-731

[7] S. Dashgupta, N. Khan, D.S.H. Pavel, A.I. Sarkar, M. Khan, "Morphological Analysis of Inflecting Compound words in Bangla", International Conference on Computer, and Communication Engineering (ICCIT), Dhaka, 2005, pp. 110-117

[8] M.N.Y. Ali, J.K. Das, S.M. Abdullah Al Mamun, A. M. Nurannabi,"Morpholoical Analysis of Bangla worfs for Universal Networking Language", icdim, 08 .

[9] Bangla Academy (2004), Bengali-English Dictionary, Dhaka.

[10] H. Uchida, M. Zhu, "The Universal Networking Language (UNL) Specification Version 3.0", Technical Report, United Nations University, Tokyo, 1998

[11] Enconverter Specifications, version 3.3, UNL Center/ UNDL Foundation, Tokyo, Japan 2002.

[12] Deconverter Specifications, version 2.7, UNL Center/ UNDL Foundation, Tokyo, JapanA.

[13] D. M. Shahidullah, "Bangala Vyakaran", Maola Brothers Prokashoni, Dhaka, August 2003,pp.110-130

[14] D. C. Shniti Kumar,"Vasha-Prokash Bangla Vyakaran", Rupa and Company Prokashoni, Calcutta, July 1999, pp.170-175

\section{AUTHORs PROFILE}

Muhammad Firoz Mridha is now a full time faculty in Stamford University Bangladesh. He obtained M.Sc. in Computer Science and Engineering from United International University (UIU) in 2010 and B.Sc. in Computer Science Engineering 2004 from Khulna University of Engineering and Technology(KUET). His research interest includes Natural Language Processing, Artificial Intelligence and Software.

Kamruddin Md. Nur attended Victoria University of Wellington, New Zealand (VUW) for his bachelor in Computer Science (2004) and United International University (UIU), Bangladesh for his masters in Computer Science (2010). He is a permanent faculty member of Computer Science department at Stamford University Bangladesh and his research interests include - Software Engineering Approaches, Software Visualization, Web Engineering, Ubiquitous and Mobile Computing, Usability Engineering, Artificial Intelligence etc.

Manoj Banik was born in Habiganj, Bangladesh in 1972. He completed his B.Sc. Engineering in Computer Science and Engineering from BUET, Dhaka, Bangladesh and M.Sc. Engineering in Computer Science and Engineering from UIU, Dhaka, Bangladesh. Now, he is working as an Assistant Professor in the Department of Computer Science and Engineering of Ahsanullah University of Science and Technology, Dhaka, 
Bangladesh. His research interests include Speech Recognition, Phonetics, Universal Networking Language and Algorithms. He is a member of Bangladesh Engineers Institute (IEB).

Mohammad Nurul Huda was born in Lakshmipur, Bangladesh in 1973. He received his B. Sc. and M. Sc. in Computer Science and Engineering degrees from Bangladesh University of Engineering \& Technology (BUET), Dhaka in 1997 and 2004, respectively. He also completed his $\mathrm{Ph}$. D from the
Department of Electronics and Information Engineering, Toyohashi University of Technology, Aichi, Japan. Now he is working as an Associate Professor in United International University, Dhaka, Bangladesh. His research fields include Phonetics, Automatic Speech Recognition, Neural Networks, Artificial Intelligence and Algorithms. He is a member of International Speech Communication Association (ISCA). 